\title{
Photon Correlations and Cross-Correlations from a Single CdTe/ZnTe Quantum Dot
}

\author{
C. Couteau ${ }^{a}$, S. Moehl ${ }^{b}$, J. Suffczyński ${ }^{c}$, J.A. GAJ $^{c}$, \\ F. Tinjod ${ }^{b}$, J.M. GÉRARD ${ }^{b}, \mathrm{~K} . \mathrm{KHEnG}^{b}, \mathrm{H} . \mathrm{MARIETte}^{b}$, \\ R. ROMESTAin ${ }^{a}$ AND J.P. POIZAT ${ }^{a}$ \\ ${ }^{a}$ CEA-CNRS-UJF Joint Group Nanophysics and Semiconductors \\ Laboratoire de Spectrométrie Physique, CNRS UMR 5588 \\ Université J. Fourier Grenoble 1, 38402 Saint-Martin d'Hères, France \\ ${ }^{b}$ CEA-CNRS-UJF Joint Group Nanophysics and Semiconductors \\ CEA/DRFMC/SP2M, 17 rue des Martyrs, 38054 Grenoble, France \\ ${ }^{c}$ Institute of Experimental Physics, Warsaw University \\ Hoża 69, 00-681 Warsaw, Poland
}

We report correlation and cross-correlation continuous wave measurements in II-VI quantum dots grown by molecular beam epitaxy. Combination of spectral selection, saturation measurements and good temporal resolution allowed us to see an antibunching effect on photons from radiative recombination of excitons in a single CdTe/ZnTe quantum dot, as well as cross-correlation within the biexciton $\left(X_{2}\right)$-exciton $(X)$ radiative cascade from the same dot. We discuss the results of our experiments in terms of a model of excitonic multitransitions.

PACS numbers: 78.67.Hc, 42.50.Dv

\section{Introduction}

When charge carriers or excitons are confined in all three dimensions in a semiconductor material, atomic-like behaviors are expected.

In particular, quantum dots (QDs) grown by molecular beam epitaxy (MBE) [1] are a typical system with such properties. In the last 10 years, an extensive study of these systems has been carried out, with in particular the possibility of 
accessing the luminescence of a single quantum dot [2], especially in self-assembled quantum dot (SAQD) systems. An important point in studies of QDs consists in using them as sources of single photons [3, 4] and/or correlated photon pairs [5]. This property of QDs is important for their possible applications in quantum cryptography [6], and in a more distant perspective, in quantum computing [7].

Most of the results on single photon sources using SAQDs have been obtained with III-V semiconductors. Only a few recent papers [8-10] present photon correlation measurements on II-VI SAQDs, all with CdSe/ZnSe. The family of II-VI materials offers several advantages for single photon emission such as greater exciton and biexciton binding energies, and shorter radiative lifetimes, making the excitons and their coherent states more robust and allowing the QDs to function at higher temperatures.

\section{Experimental setup}

The sample was grown in a molecular-beam epitaxy chamber and is made of CdTe QDs sandwiched between ZnTe barriers. After formation of the quantum islands or QDs, and subsequent capping with a layer of ZnTe, a thin mask of $100 \mathrm{~nm}$ thick aluminium was deposited on the sample with square apertures from $0.2 \mu \mathrm{m}$ to $10 \mu \mathrm{m}$ wide, allowing us to locally excite only few quantum dots. A more detailed description of the growth of the sample can be found in [11].

The sample was placed on a cold finger of a helium flux cryostat keeping the QDs at about $5 \mathrm{~K}$ for all the experiments described here. The cryostat is mounted on $X Y Z$ micrometric precision translation stages. A microscope objective (numerical aperture of 0.4 ) is used to focus a continuous wave (cw) argon laser with an excitation wavelength of $488 \mathrm{~nm}(2.541 \mathrm{eV})$ or a pulsed titanium-sapphire laser which is frequency doubled with the help of a non-critical phase matched LBO crystal at $520 \mathrm{~nm}(2.385 \mathrm{eV})$. The repetition rate is $80 \mathrm{MHz}$ with $1.2 \mathrm{ps}$ pulses. This microscope can be moved with the help of $X Y Z$ piezo drivers in order to optimize the final positioning of the laser spot onto the chosen aperture.

The photoluminescence (PL) from the dots is then collected by the same microscope and sent to a 50/50 beamsplitter (BS) for correlation measurements. In each arm after the beamsplitter, the light is dispersed by a $30 \mathrm{~cm}$ monochromator in one path, and a $50 \mathrm{~cm}$ one in the other path both with gratings of 1200 grooves $/ \mathrm{mm}$. Each spectrometer is equipped with a mobile mirror to switch the output between a CCD camera for the visualization of the spectrum or a single photon detector. In our case, microchannel plate photomultiplier tubes (MCPPMT) are used as detectors. They send electrical pulses to a time-correlated single photon module. The module is plugged into a computer and contains all the electronics necessary for the data processing.

The overall temporal resolution of our setup, essentially due to the jitter of the detectors and the dispersion of the gratings of the monochromators, was 
measured with the doubled Ti:sapphire laser at $80 \mathrm{MHz}$. A Gaussian fitting curve with a FWHM of 140 ps was found in the correlation configuration. Let us note that such a temporal resolution is not possible with avalanche photodiodes as the time jitter of about $350 \mathrm{ps}$ each is almost ten times longer than for a MCP-PMT alone. To our knowledge, our setup presents the shortest time resolution ever reported for photon correlation measurements.

\section{Excitation intensity dependence}

Before looking at any kind of correlation measurement, the first step was to observe the photoluminescence with our CCD cameras at the output of the monochromators. Figure 1 shows a spectrum of a single quantum dot from a $0.2 \mu \mathrm{m}$ wide aperture at low excitation (arbitrarily called $P_{0}$ ) for the upper graph, and at higher excitation $\left(160 P_{0}\right)$ for the lower graph. The excitonic emission was seen at $555.5 \mathrm{~nm}(2.232 \mathrm{eV})$ which is on the high energy side of the distribution of CdTe QDs. A quantum efficiency of the order of only $1 \%$ for our detectors has been found at such wavelengths. Let us note that this figure drops dramatically at slightly higher wavelengths therefore forcing us to look for QDs on the high energy side of the distribution only.

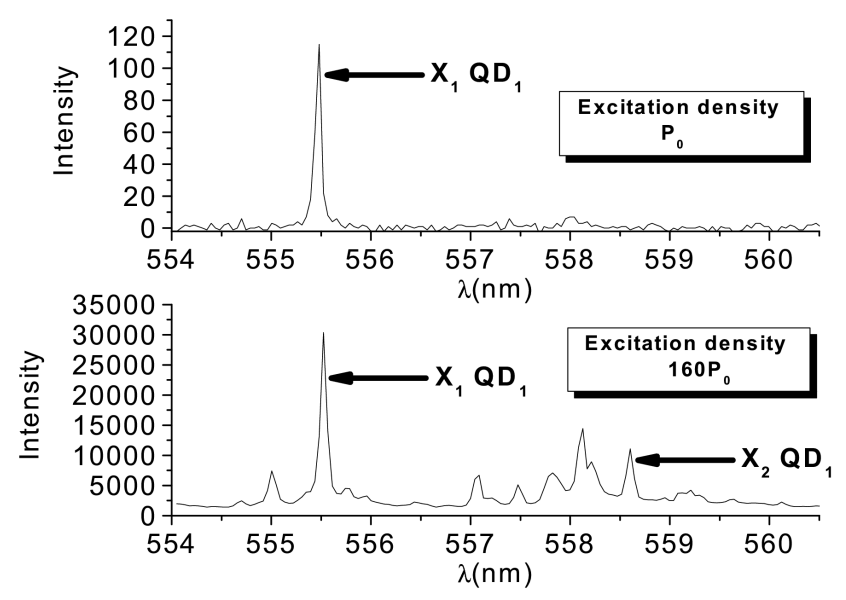

Fig. 1. Spectrum of the QD of interest for 2 different excitation intensities.

We can see in Fig. 1 that only a single excitonic line appears for low excitation and that more peaks show up as the excitation increases, especially the biexcitonic line at $558.6 \mathrm{~nm}(2.219 \mathrm{eV})$. A tentative identification of the $X_{2}$ line and its assignment to the same dot were based on relative energy positions of the two lines. It was confirmed by subsequent saturation measurements and finally proven by the observation of the cross-correlation, as described below. After having established which QD we wanted to work with (called QD1 in this paper), we 
performed lifetime measurements for the exciton and its associated biexciton using the frequency-doubled pulsed Ti:sapphire laser. The PL falls onto one MCP-PMT to start the temporal histogram measurement while a photodiode receiving the laser light directly stops it. We obtained the decay time values $1 / \Gamma_{1}=251 \pm 5 \mathrm{ps}$ and $1 / \Gamma_{2}=185 \pm 4 \mathrm{ps}$ for the exciton and the biexciton, respectively.
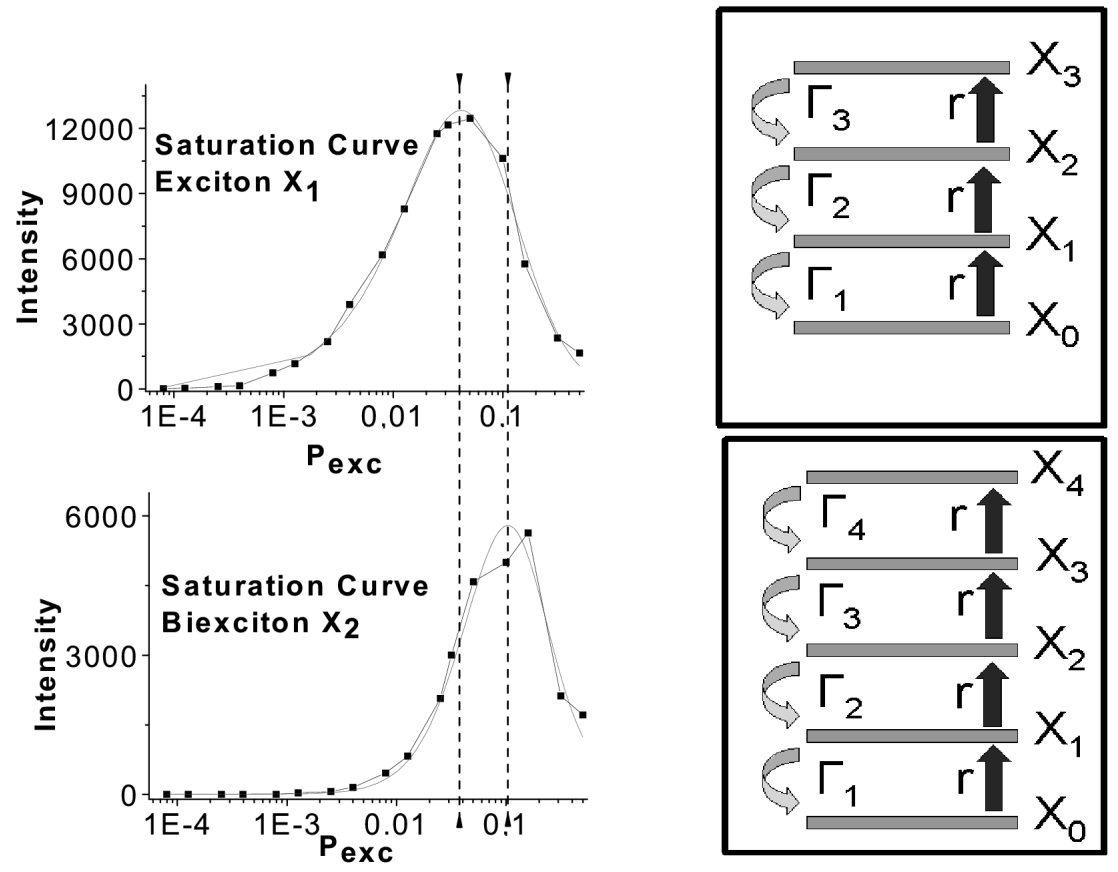

Fig. 2. Integrated quantum dot PL against excitation intensity for the exciton (upper graph) and for the biexciton (lower graph). On the right, the levels used in the ladder model to fit the data.

Next we carried out saturation measurements of the integrated quantum dot photoluminescence against excitation intensity. Figure 2 displays such results in semi-logarithmic scale, where the upper graph represents the saturation curve of the exciton of QD1 while the lower one represents the saturation curve of its associated biexciton. The solid lines on each curve are fitting functions computed using a ladder model of multiexcitons in a single QD inspired by [12]. We have taken a population of up to 3 and 4 excitons in the dot for fitting curves to the $X_{1}$ and to the $X_{2}$ respectively. Using the stationary rate equations from the model we can show that the population of $X_{1}$ and $X_{2}$ are respectively given by

$$
n_{X_{1}}=\frac{r}{\Gamma_{1}+r+\frac{r^{2}}{\Gamma_{2}}+\frac{r^{3}}{\Gamma_{2} \Gamma_{3}}},
$$




$$
n_{X_{2}}=\frac{r^{2}}{\Gamma_{2}\left(\Gamma_{1}+r+\frac{r^{2}}{\Gamma_{2}}+\frac{r^{3}}{\Gamma_{2} \Gamma_{3}}+\frac{r^{4}}{\Gamma_{2} \Gamma_{3} \Gamma_{4}}\right)},
$$

where $r$ is the pumping rate which we will henceforth be calling also the excitation parameter, and $1 / \Gamma_{i}$ is the decay time of the $i$-th exciton in the QD. Exciton and biexciton lifetimes were determined in direct measurements. For the triexciton $\left(X_{3}\right)$ and the quadriexciton $\left(X_{4}\right)$ decay times, we used values coming from integrated PL measurements versus excitation power on $\mathrm{CdTe} / \mathrm{ZnTe} \mathrm{QDs}$ from the literature [13].

\section{Photon correlations and cross-correlations}

When looking at the luminescence of a single quantum dot under cw excitation, a fall of the rate of the coincidences between the two arms of the setup is expected at zero delay, showing that the exciton of the quantum dot just recombined to create a photon and will then need a certain time before emitting another one. Figure 3 shows the autocorrelation function [14] $g^{(2)}(\tau)$ of exciton photons on the upper graph, and the cross-correlation function $g^{(12)}(\tau)$ between the exciton and its biexciton on the lower graph.

In these measurements we have performed a careful calibration of the pump power in unit of $\Gamma_{1}=1 / T_{X_{1}}$, where $T_{X_{1}}$ is the lifetime of the exciton. This has been done by fitting the luminescence intensity versus the pump power using the multiexciton ladder model presented above [12]. The autocorrelation and cross-correlation function of Fig. 3 were obtained with a QD excitation rate $r=0.65 \Gamma_{1}$ and $r=0.20 \Gamma_{1}$ respectively.

After convolution with the time resolution of our setup, the bold line in Fig. 3 upper graph is a fitting curve using the same ladder model as described above. We can see that with this physical model, there is a factor of 4 difference between the FWHM of the experimental curve and the fitting one obtained using this multitransition model. Similar discrepancies were experienced in previous studies on III-V compounds $[16,17]$. The presence of dark excitons may explain this discrepancy.

The lower graph in Fig. 3, on the other hand, shows the correlation measurement between the same exciton and its biexciton [12, 18]. For that, we used the same setup but we tuned one of the spectrometers to the wavelength of the biexcitonic line. When $X_{2}$ triggers the start and $X_{1}$ stops the time counter, a discontinuity at $\tau=0$ is expected with value above unity for $\tau>0$ revealing the bunching part of the cascade [18]. For $\tau<0$, antibunching is expected, since a recycling time is necessary for a biexciton photon to be emitted after the last exciton has been recombined. Our cross correlation result displays very clearly the bunching part for positive times, and less clearly the antibunching part for negative times. The bold line is the fitting curve using the same ladder model with up to 4 excitons in the dot. Now, we observe that, contrary to the autocor- 


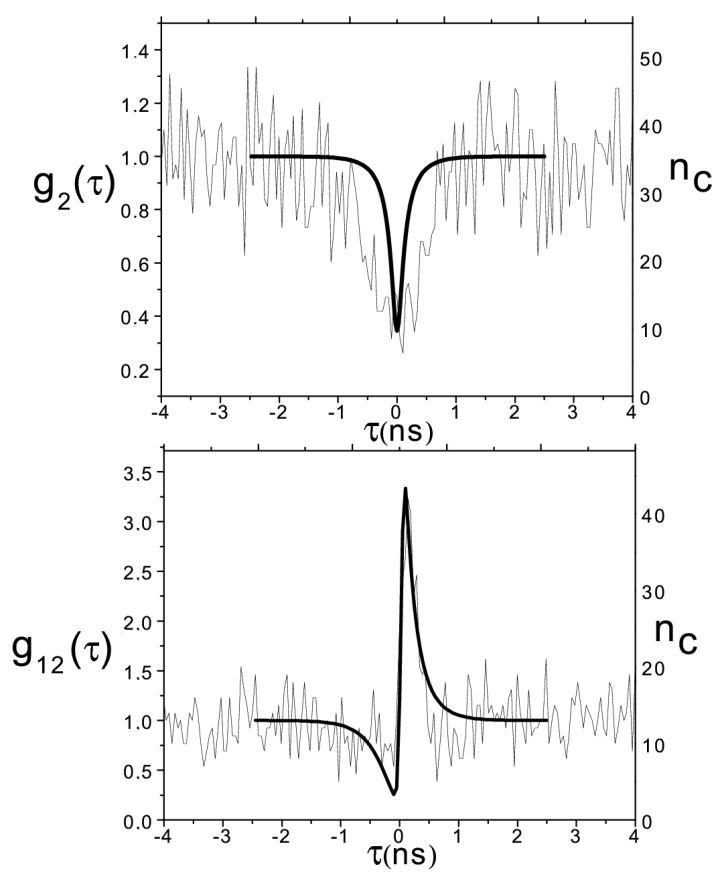

Fig. 3. Autocorrelation of exciton photons (upper graph) and cross-correlation between an exciton and its biexciton (lower graph). In both cases the right axis is the raw coincidence number $n_{\mathrm{c}}$ between the two arms ( 1 and 2 ) of the setup during the total acquisition time. The left axis corresponds to the normalized data that has also been corrected from the background noise [15]. The bold lines represent simulations obtained with multiexcitonic model (see text for details). The time bin is $49 \mathrm{ps}$ for both curves. For the autocorrelation (upper curve) counting rates were $n_{X_{1}}=10000 \mathrm{cps}$ and $n_{X_{2}}=$ $7000 \mathrm{cps}$ on a single exciton line. The dark count rate of each channel were $n_{\mathrm{d} 1}=$ $1000 \mathrm{cps}$ and $n_{\mathrm{d} 2}=800 \mathrm{cps}$. The total acquisition time was 3 hours and $30 \mathrm{~min}$. For the cross-correlation (lower curve), counting rates are $n_{2}=4800 \mathrm{cps}$ for the $X_{2}$ and $n_{1}=7600 \mathrm{cps}$ for the $X_{1}$ with background count rates of $n_{\mathrm{d} 2}=400 \mathrm{cps}$ and $n_{\mathrm{d} 1}=600 \mathrm{cps}$. The acquisition time was $9720 \mathrm{~s}$.

relation measurement, we do have the correct decay time corresponding to $T_{X_{1}}$ for positive times. The discrepancy of a factor of 4 for the exciton autocorrelation is no longer present in cross-correlation measurements. This indeed suggests an influence of dark excitons which would explain the discrepancy in the autocorrelation measurement, since in cross-correlation experiments the recombination of a biexciton always leaves an exciton in a bright state. 


\section{Conclusion}

To conclude, thanks to our temporal resolution, we presented the first $\mathrm{cw}$ autocorrelation and cross-correlation measurements of a single epitaxied quantum dot made of II-VI materials. We reported expected and unexpected behaviors from these results as discussed above. In order to have a better understanding of the temporal parameters involved in this work, further experiments need to be done, like temporal evolution of the QDs fluorescence versus temperature and quasi-resonant excitation to selectively activate one, and only one, excitonic transition.

\section{Acknowledgments}

We thank Ph. Grangier for the loan of the two microchannel plate photomultipliers, M. Terrier for the processing of the sample, and F. Donatini for experimental assistance. This work has been supported by the French Ministry for Research (ACI "Polqua", and ACI Jeune Chercheur), the "Sciences et Technologies de l'Information et de la Communication" Department of the CNRS, and State Committee for Scientific Research (Poland) (grant No. PBZ-KBN-044/P03/2001).

\section{References}

[1] L. Goldstein, F. Glas, J.Y. Marzin, M.N. Charasse, G. Le Roux, Appl. Phys. Lett. 47, 1099 (1985).

[2] D. Bimberg, M. Grundmann, N.N. Ledentsov, Quantum Dot Heterostructures, Wiley 1999.

[3] P. Michler, A. Kiraz, C. Becher, W.V. Schoenfeld, P.M. Petroff, L. Zhang, E. Hu, A. Imamoglu, Science 290, 2282 (2000).

[4] C. Santori, M. Pelton, G.S. Solomon, Y. Dale, Y. Yamamoto, Phys. Rev. Lett. 86, 1502 (2001).

[5] O. Benson, C. Santori, M. Pelton, Y. Yamamoto, Phys. Rev. Lett. 84, 2513 (2000).

[6] N. Gisin, G. Ribordy, W. Tittel, H. Zbinden, Rev. Mod. Phys. 74, 145 (2002).

[7] E. Knill, R. Laflamme, G.J. Milburn, Nature 409, 46 (2001).

[8] K. Sebald, P. Michler, T. Passow, D. Hommel, G. Bacher, A. Forchel, Appl. Phys. Lett. 81, 2920 (2002).

[9] S.M. Ulrich, S. Strauf, P. Michler, G. Bacher, A. Forchel, Appl. Phys. Lett. 83, 1848 (2003).

[10] T. Aichele, V. Zwiller, O. Benson, I. Akimov, F. Henneberger, J. Opt. Soc. Am. $B$ 20, 2189 (2003).

[11] F. Tinjod, B. Gilles, S. Moehl, K. Kheng, H. Mariette, Appl. Phys. Lett. 82, 4340 (2003).

[12] D.V. Regelman, U. Mizrahi, D. Gershoni, E. Ehrenfreund, W.V. Schoenfeld, P.M. Petroff, Phys. Rev. Lett. 87, 257401 (2001). 
[13] L. Besombes, Ph.D. thesis, Université Joseph Fourier Grenoble 2002.

[14] R.J. Glauber, Phys. Rev. 130, 2529 (1963).

[15] R. Brouri, A. Beveratos, J.Ph. Poizat, Ph. Grangier, Opt. Lett. 25, 1294 (2000).

[16] C. Becher, A. Kiraz, P. Michler, A. Imamoglu, W.V. Schoenfeld, P.M. Petroff, L. Zhang, E. Hu, Phys. Rev. B 63, 121312 (2001).

[17] E. Moreau, Ph.D. thesis, Université Paris-Sud Orsay 2002.

[18] E. Moreau, I. Robert, L. Manin, V. Thierry-Mieg, J.M. Gérard, I. Abram, Phys. Rev. Lett. 87, 183601 (2001). 